\title{
STELLAR KINEMATICS AND GALACTIC RESEARCH INVOLVING PROPER MOTIONS
}

\author{
(Invited Paper) \\ S. V. M. CLUBE \\ Royal Observatory, Edinburgh, U.K.
}

\begin{abstract}
A method of analysing proper motions as observed over all the sky is described. The kinematics of nearby stars derived from the Lick Pilot Survey are discussed, and the consequences to galactic structure examined.
\end{abstract}

The Lick Pilot Survey has provided us with a catalogue of proper motions over a wide area of the sky relative to a completely new (presumed) inertial framework. These proper motions are quite independent of the normal difficulties associated with specifying the equator and the ecliptic. Preliminary though the catalogue of data is at present, it clearly indicates that the conventional Oort-Lindblad model of stellar kinematics in the immediate solar neighbourhood is not correct (Clube, 1973). The possibility exists of course that the Lick data is vitiated by a systematic error which causes the stars to take up some spurious motion, but in spite of careful searching, there is no evidence at present for such an error (S. Vasilevskis, private communication). However, it is of some importance to note that the pattern of kinematics portrayed by the faint star proper motions bears quite remarkable resemblances to the known kinematics of intrinsically bright nearby stars - a coincidence that it would be difficult to understand were it to be attributed solely to systematic error in the proper motions. Since proper motions have in the past played a not inconsiderable role in developing our ideas regarding galactic structure and dynamics, it perhaps comes as a surprise to have it said that these recent absolute proper motions are not completely in accord with such ideas. However, the disagreement arises not so much because of any substantial difference between the Lick proper motions and those obtained previously, but more because of the use of a better kinematic model in their analysis. The precision of this model could be improved if an even greater number of absolute proper motions were available.

The great success of the Oort-Lindblad model in accounting for the main features of the kinematics of distant OB stars and cepheids (and later, neutral hydrogen) probably contributed to the decline into relative obscurity of the many papers in the 1930 's which argued the lack of generality that existed in this model. Amongst these was an important paper by Milne who indicated that to a first order, the continuous stellar velocity field expressed locally by the vector $\mathbf{v}$ at a point distant $\mathbf{r}$ from the sun, could be given quite generally by:

$$
\begin{aligned}
& \mathbf{v}=\mathbf{u}+M \mathbf{r} \\
& M=\frac{\partial u_{i}}{\partial x_{j}} \quad i, j=1,2,3,
\end{aligned}
$$


where $\mathbf{u}$ is the reflex of the solar motion and $u_{i}, x_{j}$ are velocity and distance components along (for example) the three principal galactic axes. The use of the first order Oort-Lindblad model was equivalent to setting

$$
\frac{\partial u_{1}}{\partial x_{2}}=A-B, \quad \frac{\partial u_{2}}{\partial x_{1}}=A+B
$$

and all the remaining elements of $M$ equal to zero, but it was emphasised that the real kinematic grounds for adopting the Oort-Lindblad model should have been to demonstrate statistically non-significant departures of these remaining elements from zero. This was never done, though one or two investigations gave results which implied significant departures (e.g. Mineur, 1930). In practice, the support for the OortLindblad model has come mainly from other considerations and perhaps partially from apparently acceptable values for $A$ and $B$. The chief reason it would seem for the failure to exploit Milne's theory fully was simply the computational problem of handling 12 unknowns. Comparative simplicity of analysis was preserved by keeping to five unknowns $(\mathbf{u}, A, B)$ though prior to the direct measurement of absolute proper motions, it has been necessary to carry two further rotational components $\Delta n, \Delta k$ originating from errors in precession and the motion of the equinox. Now that the computing problem no longer exists, a return to Milne's theory has long been overdue.

Of course, a lack of generality exists even in Milne's model since it does not include terms in

$$
x_{j} x_{k} \frac{\partial^{2} u_{i}}{\partial x_{j} \partial x_{k}}
$$

and other higher order terms. No analysis including such terms has yet been attempted, though there are good observational grounds for at least including

$$
x_{3}^{2} \frac{\partial^{2} u_{i}}{\partial x_{3}^{2}}
$$

in the analysis of the Lick absolute proper motions - or indeed, any others. These terms correspond to a change of secular parallax with galactic latitude regardless of sign, and although of unknown origin at present, have at least two plausible explanations, namely

(1) the Stromberg drift of high latitude faint stars with assumed larger velocity dispersion; and/or

(2) the inclusion amongst low latitude faint stars of significant numbers of very distant stars with effectively zero secular parallax.

It is unlikely however that the first order treatment of the Lick absolute proper motions will have been seriously affected by the failure to include such second order terms since the dominant effects in $M$ are parallel to the $\left(x_{1} x_{3}\right)$ plane and therefore virtually independent of changes in the secular parallax which are mostly in the general direction of galactic rotation. 
The tensor $M$ describing the velocity field of an effectively random selection of 9th magnitude stars in the Lick Pilot Survey is given by

$$
\begin{array}{rcrl}
0.22 & -0.35 & 0.90 \\
0.45 & (0.00) & 0.16 \\
-0.12 & -0.41 & -0.12
\end{array} \quad \text { "per century }( \pm 0.25 \text { approximately })
$$

The non-zero values of at least one of the elements is significant and the disagreement with the Oort-Lindblad model quite pronounced. The simplest interpretation of $M$ would attribute it to a shear of 1 ."10 about the direction $l \approx 105^{\circ}, b \approx 20^{\circ}$ with the component above the sun and mostly in the third and fourth quadrants of galactic longitude directed roughly $10^{\circ}$ below the galactic centre, and the component below and in the first and second quadrants directed $10^{\circ}$ above the anticentre. Since $M$ is in fact portraying the spatial distribution of star velocity residuals after removal of the solar motion, it is pertinent to note that the direction of this shear is parallel to the principal (deviated) axis of the velocity ellipsoid of nearby stars. But even more remarkable is the fact that the 'upper' component has an almost identical motion to that of Drift II and is in that part of the sky where Gould's Belt and the Ursa Major Stream are most conspicuous, while the 'lower' component corresponds to the motion of Drift I, and is to a large extent in that part of the sky where the Hyades group predominates. The concept of stellar drifts has today fallen into disuse, but its affinity to the idea of stellar groups is very close. It has always been difficult to understand why no counterpart to Drift II has ever been discovered amongst nearby stellar groups, none of which is moving relative to the sun towards the fourth quadrant of galactic longitude. Since Gould's Belt has the motion of the Pleiades Group and the Ursa Major Stream has that of the Sirius Group, and the average of these two motions corresponds closely to that of Drift II, it seems that the quality of early data was not good enough to permit a proper separation of Drift II into these, its two principal components. It may be concluded that the 9 th mag. stars have just that value for $M$ one would expect were they to mimic the general group properties of brighter stars both in their motions and their spatial distribution. As already suggested, it would seem hardly credible that this coincidence should occur if the properties of $M$ were in the main due to any systematic error in the Lick proper motions. It is clearly of very great importance to increase on the number of proper motions available in the present catalogue to confirm these properties of $M$.

If the reality of $M$ is accepted, the consequences to galactic structure are far reaching, and these have been discussed in an earlier paper. Suffice to say that $M$ has a rotation component about the galactic pole contrary to the direction of galactic rotation, and that this is likely to have caused serious underestimation in the past of $|B|$ especially from stars within about $300 \mathrm{pc}$ of the Sun. Correcting for the effects of $M$ from nearby stars on the more distant stars present amongst fainter magnitudes in the Lick catalogue leads to revised values for $A$ and $B$ and an indication that the Galaxy may be expanding violently. (It is presumed that the more distant stars do in fact move more in accord with the general predictions of the Oort-Lindblad model.) It is 
now very necessary to search out those observations which enable a distinction to be drawn between the conventional steady state model of the galaxy and the proposed unstable model. Perhaps this can be fairly described as 'a new problem in astrometry!'

\section{References}

Clube, S. V. M.: 1973, Monthly Notices Roy. Astron. Soc. 161, 445.

Milne, E. A.: 1935, Monthly Notices Roy. Astron. Soc. 95, 560.

Mineur, H.: 1930, Monthly Notices Roy. Astron. Soc. 90, 789.

\section{DISCUSSION}

Edmondson: I wish to add to Dr Clube's paper that I published a paper in the Monthly Notices a year or two after Milne's paper in which I extended Milne's work to second order terms. The complete expansion of the second order coefficients is given in an appendix to the paper. Subsequently in another paper in the Monthly Notices I applied this work to the interpretation of the variation of the McCormick secular parallaxes with galactic latitude.

More important, the second order effects include terms which distort the solar motion terms in the conventional solar motion and galactic rotation solution. I have discussed this in an article in Vol. 52 of the Handbuch der Physik and at a Joint Discussion at the Hamburg IAU meeting.

Murray: Have you studied the $M$ matrix for the fainter stars in the Lick Programme?

Clube: Yes. The fainter magnitude groups in the Pilot Survey are at 13th and 16th mag. photographic. After correction for the influence of nearby stars, $M$ for the more distant stars indicates that the values of Oort's constants $A$ and $B$ are numerically larger, that there is a compression in the velocity field of the wider solar neighbourhood (beyond $300 \mathrm{pc}$ ) and that the streamlines of differential motion are not parallel to the direction of galactic rotation. Elsewhere, I have tentatively interpreted this as implying a large scale expansion of the Galaxy.

Dieckvoss: In Hamburg we have in background storage data for 180000 stars of AGK3 sorted according to spectra (HD, Vyssotsky) giving magnitude (AGK2), galactic longitude and latitude, components of proper motion in galactic co-ordinates, corrected, however, for standard Oort's $A$ and $B$, and for precessional correction as recommended by Prof. Fricke. Dr Clube is invited to discuss this collection of data.

Clube: Thank you. I have in fact already examined the early type stars in the AGK3 (Monthly Notices Roy. Astron. Soc. 159, 289, 1972). These also indicate that $|B|$ has a numerically larger value than the conventionally adopted one.

Fricke: Hopf was the first who suggested a more general derivation of Oort's formulae for galactic rotation including a shear and a dilation in a velocity field, in which the mean velocity of the stars per volume element is a continuous function of the distance from the Sun. Ogorodnikov ( $Z$. Astrophys. 4, $190,1932)$, who has given essentially the same formulae, which were also derived independently by Milne in 1935, acknowledges that Hopf "suggested to him the general and elegant method and also contributed a great deal in working out the numerical results". Shatsova (Publ. Astron. Obs. Leningrad 15, 113, 1950) has applied these formulae to the proper motions in the G.C. She came to the conclusion that a local stellar system' or a cloud, in which the Sun is situated, is in rapid rotation. Obviously nobody has been able to confirm her results. In analysing the FK4 proper motions in 1967, I attempted to check her results, and I found that there can only be a very small rotation in the velocity field of the stars nearer than $300 \mathrm{pc}$. The continuation of my efforts has shown that there are clear indications of deviations from the field of galactic rotation within $300 \mathrm{pc}$. The results depend on the types of the stars which are being considered. Therefore for the application of the methods developed by Ogorodnikov and Milne one needs to know MK types and photometric data so that distances can be determined and classes selected for the study of their kinematical behaviour. For the stars further away than $300 \mathrm{pc}$ the Oort-Lindblad model of galactic rotation remains valid if one applies it to the mixture of stars in the disk populations. My recent results give no indication that the values of $A$ and $B$ which are in common use are wrong. 